\title{
Les combats catholiques dans la presse
} révolutionnaire (1789-1799)

The Catholic Struggles in the Revolutionary Press (1789-1799)

\section{Guillaume Colot}

\section{(2) OpenEdition \\ 1 Journals}

Édition électronique

URL : https://journals.openedition.org/ahrf/10719

DOI : 10.4000/ahrf.10719

ISSN : 1952-403X

Éditeur :

Armand Colin, Société des études robespierristes

Édition imprimée

Date de publication : 1 janvier 2009

Pagination : 49-71

ISBN : 978-2-200-92557-4

ISSN : 0003-4436

Référence électronique

Guillaume Colot, «Les combats catholiques dans la presse révolutionnaire (1789-1799) », Annales

historiques de la Révolution française [En ligne], 355 I janvier-mars 2009, mis en ligne le 01 janvier 2012, consulté le 23 avril 2022. URL : http://journals.openedition.org/ahrf/10719 ; DOI : https://doi.org/ 10.4000/ahrf.10719 


\title{
LES COMBATS CATHOLIQUES \\ DANS LA PRESSE RÉVOLUTIONNAIRE (1789-1799)
}

Guillaume COLOT

\begin{abstract}
L'année 1789 est une année essentielle pour le développement de la presse parisienne et provinciale et dès 1790 , des journaux affirment leur sensibilité et leur identité religieuses. Malgré la revendication contenue dans certaines appellations, il n'est pas aisé de délimiter l'ensemble des titres d'une presse que l'on pourrait qualifier de « catholique ». Les événements font émerger deux positionnements principaux : I'un en faveur des réformes, donc de la Constitution civile du clergé, et l'autre s'opposant à celle-ci, qui organise la résistance sous le Directoire et plaide pour la soumission à Rome. S'ils sont peu nombreux à consacrer l'intégralité de leur journal à la seule religion, ces journaux ne demeurent pas moins surveillés que les autres feuilles et doivent subir plusieurs fois les désagréments répressifs des autorités. Au-delà de la simple critique des lois religieuses, ils ébauchent parfois un projet de société adapté aux premières réformes et aux besoins des fidèles dans lequel croyants et religieux doivent trouver leur place. Les auteurs, anonymes ou ecclésiastiques reconvertis dans l'entreprise de presse, défendent principalement les questions doctrinales dans l'espoir de conserver ou d'attirer à eux un public séduit par leur programme dans lequel la religion prendrait toute sa place. Pour cela, les deux camps se répondent par articles interposés sur chaque point de polémique religieuse en utilisant dans leurs colonnes les mêmes procédés journalistiques : entre autres, les annonces d'ouvrages religieux, la publicité des serments ou des rétractations.
\end{abstract}

Mots-clés : presse religieuse, journaliste catholique, clergé constitutionnel, clergé réfractaire, Concile national.

La liberté d'expression accordée par la Révolution se traduit notamment par une inflation des imprimés et des écrits. Présents en tous lieux (cafés, clubs, rues, murs, Assemblée, comités, etc.), ils peuvent prendre 
toutes les formes (quotidien, périodique, pamphlet, affiche, chanson, pièce de théâtre) et touchent autant les lecteurs que les auditeurs. Selon Hegel, cité par Gérard Gengembre, la lecture devient « la prière quotidienne du monde $»^{1}$. Les journaux conquièrent un immense public car ils diffusent rapidement les informations et à un moindre coût ${ }^{2}$. Ainsi, très rapidement, tout le monde s'intéresse à la presse qui devient concomitamment un objet et un sujet politique. La presse informe, rassure, exalte, condamne, glorifie, ostracise, et souvent inquiète les gouvernements qui n'auront de cesse de l'encadrer, après un régime libéral quasi-total jusqu'en 1792, une inquiétude qui peut se lire a contrario comme un hommage rendu à l'efficacité du journal. Car, en plus de relater les comptes rendus des débats dans l'Assemblée - ce qui est le cas de l'immense majorité des journaux de 1789 -, certains mettent en avant une position idéologique de plus en plus affirmée ${ }^{3}$. La presse devient le révélateur de chaque sensibilité. L'abonné peut s'identifier à sa feuille comme à un étendard.

Dans les centaines de journaux nés en 1789 et qui rendent compte des discussions et des décrets de l'Assemblée nationale, les articles s'intéressant aux questions religieuses ne manquent pas, eu égard à l'importance des débats puis des décisions auxquels elles donnent lieu. Au même moment apparaît une presse qui, en choisissant un titre, en écrivant de longs articles ou en y consacrant l'intégralité de ses colonnes, s'intéresse exclusivement à la religion. Pourtant, malgré la revendication contenue dans certaines appellations, il n'est pas aisé de les identifier en tant que « journaux religieux » et plus encore comme « journaux catholiques ». Les inventaires réalisés par les historiens ${ }^{4}$ se limitent aux clivages politiques habituels sans définir les caractéristiques d'un « journal religieux ».

(1) Gérard Gengembre, À vos plumes citoyens, Paris, 1989, p. 10.

(2) Claude Béranger, Jacques Godechot, Pierre Guiral et Fernand Terrou, Histoire générale de la presse française, 5 tomes, Paris, PUF, 1969.

(3) Les inventaires des journaux révolutionnaires dressés par les historiens du XIX ${ }^{\mathrm{e}}$ siècle classent les journaux en fonction des idées et des mots d'ordre politiques qu'ils défendent ou qui les rassemblent : une presse « patriote » et une presse « royaliste ».

(4) Eugène Hatin, Histoire politique et littéraire de la presse en France (1859) et la Bibliographie historique et critique de la presse périodique française (1866) ; Maurice TouRnEux, Bibliographie de l'histoire de Paris pendant la Révolution française, 1890-1913, les tomes II et III sont consacrés à la presse parisienne ; Gérard WALTER, Catalogue de l'histoire de la Révolution française, Paris, Bibliothèque nationale, 1943, le tome V traite des journaux et des almanachs ; Claude BÉrANGER, Jacques Godechot, Pierre Guiral, Fernand Terrou, op. cit. ; Gilles Feyel, La presse en France des origines à nos jours. Histoire politique et matérielle, Paris, Ellipses, 1999 et Dictionnaire de la presse française pendant la Révolution, 1789-1799, la presse départementale, tome 1, Ferney-Voltaire, Centre international d'étude du XVIII, 2005. 
Comment reconnaître un tel journal parmi ceux parus en 1789 et tout au long de la période révolutionnaire ? Doit-il consacrer l'intégralité de ses numéros à la religion et notamment à la religion catholique ? Se contentet-il de rappeler les préceptes religieux sans en débattre ? Analyse-t-il, à la manière d'un journal d'information, les changements opérés dans le premier ordre depuis 1789 ? S'adresse-t-il à tous les lecteurs ou cible-t-il les seuls croyants ? Se mobilise-t-il pour la défense du clergé, de ses membres ou des fidèles ? Est-ce une presse de combat qui joint la réflexion à des actions sur le terrain?

Autant d'interrogations qui nous amènent à cerner les raisons qui ont poussé les rédacteurs à proposer des feuilles qui s'intéresseraient d'abord aux questions religieuses parmi les nombreux autres débats abordés par la presse parisienne. Fort de ce questionnement, nous avons choisi de commencer par l'étude des titres, qui ne sauraient être décidés au hasard mais comme emblématiques d'un contenu : ils offrent aux lecteurs une facilité classificatoire ${ }^{5}$ et permettent de trouver dans les inventaires concernant le très vaste corpus journalistique ${ }^{6}$, une presse à dominante « religieuse ». Nous avons conscience des limites de ce choix méthodologique pour un premier défrichement, mais le titre permet au lecteur d'identifier le plus souvent fidèlement la nature du journal du moins jusqu'en l'an II. Néanmoins, à partir de l'an IV, et surtout après le coup d'État de fructidor en l'an V, le titre est moins représentatif de son parti. En effet, si chaque camp possède une tribune et sa presse associée, la censure oblige bien souvent des fusions entre deux journaux ou des changements de titres pour éloigner les foudres du Bureau des journaux du ministère de la Police générale ${ }^{7}$. La méthode de recherche des journaux participant d'un combat religieux s'apparente alors davantage au tissage d'une toile d'araignée. Un journal mentionne et salue dans ses colonnes l'esprit de feuilles qui défendent les mêmes opinions que lui, et en même temps, il proscrit dans d'autres articles les journaux irréligieux, incrédules

(5) Henri Duranton, « Les nouvelles de l'Assemblée nationale : l'enseignement des titres de presse », p. 205-214, dans Claude Labrosse et Pierre Retat (dir), La Révolution du journal, 17881794, Lyon, CNRS, 1989.

(6) Les centaines de journaux qui apparaissent dès 1789 nous offrent un immense corpus qui est conservé pour l'essentiel à la Bibliothèque nationale, aux Archives nationales, dans les dépôts d'archives départementales ou municipales et dans les bibliothèques privées.

(7) AN F7 3448 (lettres, rédacteurs, registres, abonnement, police des journaux). Les séries AN F7 (police générale), AFII (Conseil exécutif provisoire et Convention, Comité de salut public), AFIII (Directoire exécutif) fournissent des preuves de la volonté de l'État de contrôler la presse et les journalistes. Ceux qui choisissent d'écrire pour la religion n'y échappent pas. 
et trop révolutionnaires à son goût. Après les avoir repérés, il faut les sonder pour constater leur intérêt concernant les débats strictement religieux à différents moments de la période révolutionnaire. Ces tâtonnements permettent ainsi de combler au mieux les notices parfois incomplètes des inventaires des journaux conservés à la Bibliothèque nationale.

Parmi les feuilles ainsi ciblées grâce aux titres et aux références explicites des journalistes, deux positionnements principaux émergent : l'un en faveur des réformes, l'autre s'opposant à celles-ci, notamment sous le Directoire, en défendant la soumission à Rome. Il est intéressant de s'interroger, tout d'abord, sur l'existence d'une " presse religieuse et catholique » au début de la période révolutionnaire et sur son évolution, notamment à partir de 1790-1791, période au cours de laquelle elle est confrontée à une nouvelle loi religieuse (la Constitution civile du clergé). Nous découvrirons alors les idées défendues et les actions menées par les journalistes « catholiques » de 1789 à 1799.

\section{Les débuts d'une presse religieuse et catholique}

Entre 1789 et 1792, sur près de 200 titres, nous en trouvons 23 répondant à notre premier critère ${ }^{8}$. On peut imaginer avant de lire son contenu, qu'une presse s'intéresse à la religion au milieu des nombreux autres journaux qui plébiscitent les comptes rendus des débats parlementaires. Cet intérêt pour la religion n'est pas inédit car on connaît l'omniprésence de celle-ci sous l'Ancien Régime et encore pendant les premières années de la Révolution. Il n'est donc pas anormal que certains journalistes - issus ou non des cercles littéraires, hommes de lettres, philosophes, ou hommes nouveaux - chrétiens croyants et/ou pratiquants, lui consacrent tout ou partie dans leurs feuilles. Dans ces titres, les mots les plus usités concernent les « apôtres » (Actes des Apôtres, Disciples des Apôtres, Actes des bons Apôtres, Les Quatre Évangélistes...), la vision providentielle ou malheureuse de l'existence du chrétien est présente avec Le Journal du diable, Actes des martyrs. Enfin, les textes sacrés sont cités avec Le Catéchisme républicain, La Bible d'à présent, Le Catéchisme de Jean Bart, Les Annales religieuses ou le Journal ecclésiastique. Le choix de ces titres est ambigu : il peut renvoyer à l'inconscient des journalistes, qui ressentent les changements apparus en 1789 à travers le double filtre

(8) En annexe : Liste des journaux possédant dans leur titre, un mot ou une expression se rapportant à la religion. Parfois, on remarque la mention du statut social du rédacteur. On notera l'absence quasi-totale de journaux religieux en 1793 et en l'an II. 
de la religion et de la citoyenneté. Les rédacteurs prennent acte des nouveaux droits et ils les utilisent en conservant leur identité religieuse. La religion reste un guide pour apprécier les transformations futures. Elle les dirige vers des temps nouveaux, mais à la lecture des premiers titres, on ressent une appréhension à peine dissimulée sur l'avenir. Pourtant, parmi les journaux examinés, les auteurs n'utilisent pas leur liberté d'expression pour faire du prosélytisme ou pour montrer leur attachement personnel à leur foi, ils fixent leur croyance dans la sphère privée et se présentent avant toute chose comme "journalistes citoyens » plutôt que comme chrétiens. Mais il faut se méfier du titre, il n'est pas toujours ce qu'il prétend. Un titre un peu mystérieux ou énigmatique, reprenant des références culturelles communes, partagées et reconnues par l'ensemble de la population (la Bible, un apôtre...), peut participer d'une stratégie d'individualisation parmi le flot des journaux, qui demeurent avant toute chose des concurrents commerciaux dont il faut se distinguer?

Comment définir (avec ou sans le titre) les journaux « religieux »? Le ton et le style du rédacteur donnent ce qu'on appellerait aujourd'hui la « ligne éditoriale ». Parfois, la mention d'un auteur connu, à défaut de devenir immédiatement célèbre, peut aussi influencer le lecteur sur le choix de la feuille à acheter ou à lire (on pense à l'abbé Barruel ou à l'abbé Sicard, qui sont devenus postérieurement des grandes figures du journalisme). Entre 1789 et 1792, nous avons recensé seulement 5 journaux ${ }^{10}$ qui consacrent l'intégralité de leurs articles à la seule cause religieuse. Les autres journaux réagissent dans l'instant en suivant les débats de l'Assemblée nationale constituante et sont à l'écoute des modifications religieuses. Ils font des «comptes rendus ». L'analyse des premiers numéros de ces journaux ne montre pas, globalement, de plan défini, destiné à défendre la religion en tant qu'institution ou ses membres en tant qu'ordre social. Par ailleurs, les journaux « religieux » n'apparaissent pas - sauf exception - avant l'automne et le début de l'hiver 1789 (4 journaux dont Les Nouvelles ecclésiastiques qui existent avant 1789), période à partir de laquelle le conflit entre la Révolution et l'Église se met en place. Les journalistes suivent l'ordre du jour et perçoivent les premières contradictions entre la liberté générale et l'exercice individuel de la foi.

(9) Henri Duranton, op. cit., p. 205-214.

(10) Les Nouvelles ecclésiastiques, le Journal chrétien, le Journal de Bienfaisance ci-devant l'ami des vieillards, Les loisirs d'un curé déplacé ou les Actes de l'Église constitutionnelle et le Journal ecclésiastique. Il est difficile de les comparer car ils ne publient pas tous en même temps et pendant la même durée. Ils seront plus nombreux après 1794. 
À l'exception d'un journal appelé Le Juif errant ${ }^{11}$, les premiers journaux religieux sont catholiques. Ils sont rédigés par des catholiques pour des catholiques, c'est-à-dire dans leur esprit, pour la majorité des Français. La plupart regardent les réformes politiques progressives comme nécessaires, ils l'affirment dans les discours et l'écrivent dans leurs articles. C'est le cas par exemple de l'Almanach de l'abbé Maury publié de Coblentz, qui reconnaît en 1790 la nécessité de "réformer » mais sans tout détruire ${ }^{12}$. Le Journal ecclésiastique de l'abbé Barruel consacre une dizaine d'articles entre mai et décembre 1789 à la réforme nécessaire du clergé : c'est notamment le cas lorsqu'il évoque des bénéfices "détournés de leurs propres usages $»^{13}$. Antoine Rivarol condamne « l'énorme fortune du haut clergé [qui] était aussi depuis longtemps un objet insupportable aux yeux du peuple $\gg{ }^{14}$. Ce sont des thèmes également repris par des journaux plus proches du camp patriote, notamment Le Tonneau de Diogène ou les Révolutions du clergé qui donne un " coup d'œil sur l'état actuel du clergé » dans lequel il annonce, dans son prospectus, que « la partie saine du clergé a tout à gagner de la Révolution, le haut clergé et le moyen clergé ont, il est vrai tout à perdre $»^{15}$. Les critiques portent sur certains problèmes abordés par les cahiers de doléances et sur les premières décisions prises pendant l'été 1789 : la perte des privilèges de l'ordre, la disparition de la dîme et plus tard des vœux, la lutte contre les nouvelles mœurs qui peuvent conduire à l'impiété, la défense du monopole religieux. On remarque chez eux un souci de rappeler aux lecteurs que les catholiques doivent rester les principaux acteurs de leur évolution, tout en reconnaissant (même à demi-mot) les actes accomplis et notamment le transfert de la souveraineté nationale.

Les articles sont rarement signés, mais ceux qui dévoilent librement leur identité sont en majorité des ecclésiastiques ${ }^{16}$. Sensibilisés par une volonté de régénération sans remettre en cause la foi en tant que telle, les

(11) Le Juif errant, $3 \mathrm{n}^{\circ}, \mathrm{BNF} 8^{\circ} \mathrm{Lc} 22346$.

(12) L'Almanach de l'abbé Maury ou réfutation de l'Almanach du Père Gérard, couronnée par la Société des Amis de la monarchie....... à Coblentz, BNF $8^{\circ} \mathrm{Lc} 2359,1^{\mathrm{er}}$ entretien, p. 33.

(13) Le Journal ecclésiastique, mai-septembre 1789 à décembre 1790, 4 volumes, in- $8^{\circ}$, BNF H88 10 à 20.

(14) Antoine Rivarol, Mémoires ou Tableau historique et politique de l'Assemblée constituante, 1797, Paleo, Sources de l'histoire de France, p. 75.

(15) Le Tonneau de Diogène ou les Révolutions du clergé, 1790, BNF $8^{\circ} \mathrm{Lc} 2311$ (A), p. 3.

(16) Par exemple, l'abbé Barruel (Le Journal ecclésiastique) ; les abbés Sicard et Boulogne (Annales religieuses, politiques et littéraires puis Annales catholiques); L'abbé Royou (L'Ami du Roi) ; L'évêque métropolitain Le Coz (Journal du Concile national de France) ; L'abbé Grégoire (Les Annales de la religion). 
membres du clergé, conscients de certains dysfonctionnements, critiquent et souhaitent des mutations. L'abbé Barruel formule même une prière pour résoudre les problèmes de Dieu et du $\mathrm{roi}^{17}$. Les journaux plus proches du côté " patriote » pensent avant tout à réformer le fonctionnement du clergé (réduire le nombre de paroisses, faire la chasse aux bénéfices inutiles $)^{18}$ mais l'idée de détruire l'Église ou la religion est absente de leurs articles. On distingue dans les premiers écrits des journalistes catholiques, un respect du culte et des conditions matérielles de celui-ci, surtout lorsque l'Assemblée nationale s'attaque à la propriété19. Par ailleurs, en parcourant le courrier des lecteurs, on peut lire des plaintes nombreuses sur la vente des biens du clergé qui entraîne l'appauvrissement du clergé et l'enrichissement des patriotes, coupables de profiter de leur nouvelle position. D'autres lecteurs se réjouissent de cette redistribution des richesses présentant déjà un courant gallican et janséniste qui triomphera bientôt. Enfin, relayant les interventions des ecclésiastiques La Fare et Dom Gerle (en février et avril 1790), plusieurs journalistes (dont l'abbé Barruel) s'accordent à reconnaître la religion catholique comme la religion de la majorité de la population, c'est-à-dire, la religion nationale ou religion d'État. La plupart ne sont d'ailleurs pas heureux de la proclamation sur la liberté des opinions religieuses décidée pendant l'été et voient dans ce droit une dérive anarchique inéluctable ${ }^{20}$. Cependant, au milieu de l'année 1790 , on assiste à une première rupture dans la presse " catholique $»^{21}$. Alors que les journaux consacraient une place minime à la cause religieuse, celle-ci prend peu à peu plus de place dans leurs colonnes sous la forme de rubriques spécifiques («Affaires religieuses», «Loi religieuse », " Nouvelles ») ou occupe l'intégralité du journal.

\section{L'origine du « schisme » éditorial (automne 1790-1791)}

Les décrets « offensifs » adoptés depuis l'automne précédent provoquent un repli des journalistes catholiques vers la défense du « trône et de l'autel »; ils reçoivent l'aide des journaux non religieux se disant

(17) Le Journal ecclésiastique, mai 1789, p. 87-89.

(18) Le Tonneau de Diogène ou les Révolutions du clergé, 1790, n 5, p. 128.

(19) Jacques Le Goff et René RÉmOnd (dir), Histoire de la France religieuse, Paris, PointsSeuil, [1991] 2001, tome 3, p. 75.

(20) Le Journal ecclésiastique, mai 1789, p. 94 : « la multiplicité des cultes dans un état est une irreligion publique $\gg$.

(21) Michel Vovelle évoque « un tournant majeur », Timothy Tackett souligne « la première fracture » et Bernard Plongeron parle « de rupture consommée », ou « un séisme ». 
« ami du roi » (royalistes) dans leur lutte contre les effets de la nouvelle loi religieuse (la Constitution civile du clergé) votée et sanctionnée par le roi en juillet 1790. La Constitution civile du clergé ne provoque pas l'augmentation du nombre de journaux religieux, mais elle les divise : d'une part, le camp « catholique traditionnel » (défendant la primauté de Rome et du pape sur l'Église de France) et d'autre part le camp «catholique patriote » (défenseur de l'Église gallicane et de la Constitution civile) ${ }^{22}$. Entre 1789 et 1792, le premier reçoit le soutien des journaux royalistes ${ }^{23}$, dans la défense du «trône et de l'autel ». Les deux thèmes sont inextricablement liés, davantage encore depuis 1789 quand les problèmes de l'État ont pénétré les affaires religieuses. La destruction de l'un peut entraîner la disparition de l'autre.

L'objet de leur première intervention est le décret du 2 novembre 1789 sur la mise à la disposition de la nation des biens des ecclésiastiques. Les journalistes traditionnels évoquent la «ruine» du clergé. Ce qui devait être le fruit d'une démarche pragmatique pour résoudre les difficultés financières du royaume offrait aux députés une solution pour faire face aux besoins financiers de la monarchie. Les Actes des Apôtres y consacrent $13 \%$ de leurs articles, L'Ami du roi $7 \%$ et Le Journal ecclésiastique $5 \%$. Tout en s'interrogeant sur le véritable propriétaire des biens (la Nation ou l'Église), les journaux portent leurs critiques sur deux aspects de cette réforme : le premier est que le clergé ne pourra plus subvenir aux besoins des pauvres gens. Le Journal ecclésiastique pose clairement la question : «Qui s'occupera des pauvres et des prêtres ?»"

(22) Le camp « catholique patriote » est représenté par les journaux suivants : Le Journal du diable (1790), Les Actes des bons apôtres, journal des disciples de la Trinité française (1790), Le Journal des ecclésiastiques constitutionnels (1792), Correspondance religieuse et morale avec les départements (1792), Le catéchisme républicain ou la France sauvée (an II), Le Journal chrétien, puis Journal de l'Église constitutionnelle de France (1792), Les Annales de la religion (1794-1800), Le Journal du Concile national de France (1797), Le Journal du citoyen (partie politique des Annales de la religion, 1797-1798), Le Journal religieux ou Mémoires pour servir à l'histoire de l'Église (1798). Le camp «catholique traditionnel» sera défendu par les journaux suivants : Le Journal ecclésiastique (1790), L'Almanach de l'abbé Maury (1790-1791), La Bible d'à présent puis ou des septante (1790), Les quatre évangélistes ou supplément aux Actes des Apôtres (1790), Les loisirs d'un curé déplacé ou les actes de l'Église constitutionnelle (1791), A deux liars, à deux liars, le journal (1791-1792), Le Journal de bienfaisance, ci-devant l'ami des vieillards (1791), Le Journal de la religion et du culte catholique (1795), Les Annales religieuses, politiques et littéraires (1796), Les Annales catholiques (1796-1797), Les Éphémérides politiques, littéraires et religieuses (1796-1797), L'Ami de la religion, des mœurs et des sciences (1797), Le précurseur du messie (1799).

(23) Pour les plus célèbres de ces journaux « royalistes » et « catholiques » : L'Ami du roi de l'abbé Royou, La Gazette de Paris de Rozor, Le Journal de Suleau, Le Journal général.

(24) Le Journal ecclésiastique, octobre 1789, p. 195. 
Mais c'est surtout le sort réservé aux ecclésiastiques qui inquiète la « presse catholique » traditionnelle : L'Ami du roi évoque leur pauvreté et dresse des portraits de religieuses et de religieux jetés sur les routes après le décret du 13 février 1790 interdisant les vœux et supprimant les ordres religieux. Le journaliste du Disciple des Apôtres dénonce les «profiteurs », les « pillards » et les « capitalistes » qui s'enrichissent sur le dos de l’Église ${ }^{25}$.

Les journaux « religieux » patriotes y voient aussi une occasion de s'interroger sur la propriété des biens du clergé. Assurément, les journalistes véhiculent un courant d'anticléricalisme né dans les dernières décennies du siècle, qui envisageait de mieux utiliser les biens confiés aux clercs pour l'exercice de leur mission caritative et éducative. De ce fait, c'est le règne de la $\operatorname{loi}^{26}$ qui permettrait de régler les abus et de mieux répartir les richesses conformément aux cahiers de doléances ${ }^{27}$, ce qu'affirme Le Journal chrétien dans son prospectus ${ }^{28}$. Pour les journaux de l'autre camp, cette loi prouve au contraire toute son incohérence : à travers l'institution révolutionnaire, la loi n'a pour eux qu'une place secondaire, le gouvernement ne peut se substituer à l'Église au nom de la volonté générale. Les journalistes ont conscience que cette première réforme peut entraîner la chute de l'ensemble de l'édifice et ne cesseront de montrer l'usurpation des compétences des Assemblées révolutionnaires face au pouvoir spirituel. Rivarol, le journaliste qui collabora aux Actes des Apôtres, déclare dans ses Mémoires que les révolutionnaires avaient un plan préparé dans lequel il faut « d'abord révolter les peuples contre la religion et ensuite contre l'autorité $»^{29}$. Dans ces termes, on distingue la thèse du complot contre lequel il faut lutter. Les journalistes catholiques doivent-ils obéir à la loi civile quand elle est contraire à celle de la religion? Les journaux qui apparaissent en 1790 tentent de concilier la nouvelle alliance : Le Journal de bienfaisance, ci-devant l'Ami des vieillards qui paraît à l'automne 1791 propose un sous-titre : «LE ROI, LA RELIGION »", ou encore Le Journal de la noblesse, de la magistrature, du sacerdoce et

(25) Le Colporteur national dédié aux Français patriote ou Le Disciple des Apôtres, octobre 1789, BNF 8 Lc2 275.

(26) Albert Soboul, La civilisation et la Révolution française, Paris, Arthaud, 1985, p. 85.

(27) Michel Vovelle, « La politique religieuse de la Révolution française », p. 65-94, dans Jacques Le Goff et René Remond (dir), op. cit.

(28) Le Journal chrétien, p. 5, prospectus. La Constitution civile du clergé fait « d'un esclave de la religion chrétienne un citoyen » et le « plus pur appui des lois de la liberté ».

(29) Antoine Rivarol, op. cit, p. 67.

(30) Le Journal de bienfaisance, ci-devant l'Ami des vieillards, 1791, BNF 8 Lc2 616, n 38. 
du militaire qui suggère une double profession de foi : « Une foi, une loi, un roi » et $«$ DIEU et HONNEUR ${ }^{31}$. L'incursion de la politique dans la religion ne provoque pourtant pas l'organisation d'un « parti » catholique et les historiens de la presse et de la religion ne l'évoquent pas plus. On remarque ponctuellement, en fonction des événements et des débats concernant le clergé, des journalistes qui ont choisi de défendre le roi et la religion et de ne pas abandonner ce terrain à la presse patriote gouvernementale. Les attaques contre l'Église et le clergé permettent de compter ses alliés et de confondre ses ennemis. En 1792, Le Courrier du Midi prévient les lecteurs que « la plupart des journalistes sont gangrenés » et il cite les journaux suivants : «Le Logographe, Le Postillon de la guerre, L'Ami du roi, La Gazette de Paris, le Journal de Paris et la Gazette universelle peuplés de coquins $\rangle^{32}$. Les lois religieuses ont selon le mot de Rivarol des « suites et des effets sur la Révolution », sur l’Église et sur les hommes $^{33}$, et désormais jusqu'en 1799 et au fur et à mesure de l'avancée des travaux des différentes assemblées, le fossé ne cessera de se creuser entre la «presse catholique » traditionnelle et la «presse catholique » patriote.

\section{La Constitution civile du clergé (12 juillet 1790)}

Tout au long du XIX ${ }^{\mathrm{e}}$ siècle, l'historiographie catholique ne cesse d'anathématiser ceux qui ont porté et défendu cette Constitution, notamment en s'appuyant sur les écrits de l'abbé Barruel ${ }^{34}$. Délaissant progressivement la défense du « trône », les articles concernant la religion passent à $10 \%$ dans La Gazette de Paris, ils représentent les $3 / 4$ des chroniques de L'Ami du roi pendant l'été 1790 , et concernent près de $80 \%$ des articles du Journal chrétien en 1791. De cette Constitution découlera un schisme dans le clergé français et dans la presse révolutionnaire jusqu'en 1799. La Constitution civile répondait à un désir de rationalisation et à une volonté affirmée dans le clergé de revenir à la discipline de l'Église primitive.

(31) Le Journal de la noblesse, de la magistrature, du sacerdoce et du militaire, 1791, vol. 1, prospectus, BNF 8 Lc2 492.

(32) Le Courrier du Midi, 1792, BNF 4 Lc3 9, n 97, p. 390

(33) Antoine Rivarol, op. cit, p. 244.

(34) Augustin BARruEl rédige Le Journal ecclésiastique entre 1788 et 1792. D'abord favorable aux idées démocratiques, il s'oppose à la Constitution civile du clergé et s'exile à Londres. Il écrit son Histoire du clergé pendant la Révolution (1793) qui dénonce la persécution religieuse, puis ses Mémoires pour servir à l'histoire du Jacobinisme, qui paraissent en quatre volumes entre 1797 et 1799 . 
C'est une idée née au XVIII ${ }^{\mathrm{e}}$ siècle de penser que les lois sont toutes puissantes pour réformer et transformer l'ordre social. Le député Camus, au nom des membres du Comité ecclésiastique avait déclaré le $1^{\text {er }}$ juin 1790 qu'ils avaient « le pouvoir de changer la religion ». Il faut y reconnaître la victoire du gallicanisme et du « richérisme » des curés puisque désormais les circonscriptions ecclésiastiques correspondent aux découpages administratifs (autant de diocèses que de départements), et que les évêques et les curés sont élus par les citoyens «actifs " (même les non catholiques) de la paroisse et du département. Mais c'est surtout l'investiture des évêques qui fit couler de l'encre, puisque les prérogatives du pape et du roi disparaissaient.

Usant de mêmes rouages dans leurs articles, les journaux aident « leur » clergé. Les rubriques concernant la religion sont plus nombreuses et plus longues, notamment celles qui s'intitulent «Annonces » et qui sont placées systématiquement en excipit. L'annonce des livres parus ou à paraître participe activement à la controverse ${ }^{35}$; en se répondant sur un thème délicat ${ }^{36}$, ces textes permettent aux journaux de tester leur force de persuasion. Chaque rédacteur tente, dans des droits de réponse élaborés et très documentés - et souvent difficiles à lire - de montrer les erreurs de l'autre camp, en adoptant la posture d'un didacticien. En écrivant des «Apologies », des « Guides » ou des «Catéchismes » ${ }^{37}$, les journalistes dévoilent la même formation intellectuelle (le droit) et culturelle (bourgeoise). Ils privilégient la méthode inductive, ouvrant la feuille par le témoignage d'un lecteur qu'aborde un thème de controverse (le célibat des prêtres, le serment, le divorce, le mariage, le découpage des évêchés, l'investiture des prêtres...). La démonstration qui suit met en scène les acquis du passé (conciles) et les sciences de la religion (théologie, droit canon). Puis, en fin de journal, ils mentionnent les actions vertueuses ou impies des « chrétiens ». Pourtant, s'ils usent de la même méthode, nous ne remarquons pas de concertation entre les journaux d'un même camp, la seule évolution notable se trouve dans le titre qui est en prise directe

(35) Le Journal chrétien présente 43 livres, Le Journal ecclésiastique 56 pendant deux années de parution.

(36) Le 18 décembre 1791, L'Ami du roi annonce la parution d'un ouvrage intitulé « Remontrances des ecclésiastiques non assermentés à M.M les députés de l'Assemblée nationale ». Le Journal chrétien lui répond le 21 décembre (trois jours plus tard) par un «Catéchisme sur la Constitution civile du clergé ».

(37) Nouvelle apologie du clergé catholique de France contre les calomnies [...] sur les projets relatifs aux troubles religieux (1792), BNF Lc4 3874 ; Le catéchisme du curé Meslier, 1790, BNF NUMM 46484. 
avec l'actualité : Le Journal chrétien, L'Ami des mours, de la vérité et de la paix de Pierre-Vincent Chalvet devient en février 1792 Le Journal de l'Église constitutionnelle réuni au Journal chrétien ou l'ami des mours, de la religion et de l'égalité, un titre plus lisible et officiel, sans doute pour aider le clergé constitutionnel chahuté dans les départements, puis, en mai 1792, il s'intitule uniquement Journal de l'Église constitutionnelle de France. On pense également aux Annales religieuses, politiques et littéraires des abbés Sicard et Jauffret qui changent de nom à partir du numéro 21 (les Annales catholiques) peut-être pour se démarquer des Annales de la religion ${ }^{38}$. D'autres prévoient d'augmenter le nombre de pages ou de changer leur périodicité devant la nécessité des débats sur les problèmes religieux.

\section{La presse au service des combats catholiques}

Désormais s'affichent deux mondes que tout oppose, sur la place de la religion dans la société mais également sur la nature de la citoyenneté pour un chrétien. Cette nouvelle société, que tous se hâtaient d'appeler de leurs vœux, doit se bâtir sur une définition du statut du chrétien-citoyen et ses libertés dans une société laïcisée et sur la construction d'une religion nationale. Le clergé constitutionnel, éclairé par les écrits des Lumières et la présence de jansénistes dans le Comité ecclésiastique propose la régénération de la société chrétienne dans laquelle le statut des ecclésiastiques serait harmonisé. Le gouvernement aurait en charge de poser les fondements d'une religion civile et nationale (ou naturelle) qui réunirait le spirituel et le temporel. Le clergé insermenté utilise le même langage patriotique, mais pour l'Église universelle catholique, apostolique et romaine, rien ne peut la séparer de sa fidélité à Rome. De 1790 à 1799 , plusieurs thèmes cristallisent leur opposition, qui est retranscrite dans leurs journaux.

\section{L'autorité civile contre l'autorité romaine}

Les journaux «constitutionnels » défendent un héritage gallican dans lequel l'État («l'autorité civile ») est compétent par ses lois pour s'occuper des affaires spirituelles et marque sa supériorité sur l'Église de

(38) Guillaume CoLot, L'Église et la foi dans la presse catholique en Révolution, Master 2, Clermont-Ferrand, juin 2006. Trois journaux sont particulièrement étudiés : Les Actes des Apôtres, Le Journal chrétien, Les Annales religieuses, politiques et littéraires. 
France et « l'autorité romaine » du pape. Les journalistes doivent prouver aux lecteurs que l'Assemblée nationale est qualifiée pour s'occuper des questions religieuses. Le 31 août 1791, le Journal chrétien affirme « offrir aux simples, un préservatif contre les décrets abusifs de la Cour de Rome » et que « l'Église étant renfermée dans l'État et non l'État dans l'Église, est soumise à sa constitution pour le temporel $\aleph^{39}$. Le journal diffuse la troisième édition du "Préservatif contre le schisme » chez Leclerc, libraire rue Saint-Martin près celle aux Ours, $n^{\circ} 254$. En 1795, pendant les travaux préparatoires au Concile national organisé par le clergé constitutionnel, les évêques rappellent la loi absolue : «Une église gallicane [nationale] a tous les pouvoirs nécessaires pour se constituer, se gouverner, changer et améliorer sa discipline particulière, tout en respectant le dogme général $»^{40}$. Les historiens y reconnaissent la voix du jansénisme qui défend la tradition des droits français contre l'ultramontanisme ${ }^{41}$.

Pour les journaux «traditionnels » cette vision est faussée. Avec leurs collègues royalistes, ils dénoncent depuis longtemps le thème du complot (des philosophes, des jansénistes, des protestants et des francsmaçons) et l'usurpation des pouvoirs par les députés de l'Assemblée nationale ${ }^{42}$, coupables de porter tous les maux et vices. Dès février 1790 et pendant les discussions sur le futur texte, le Journal ecclésiastique s'interroge si « l'Assemblée nationale a le droit d'établir, de détruire ou de réduire les évêchés ? » en rappelant qu'il existe une distinction entre les pouvoirs politiques et ecclésiastiques. Contrairement à la Constitution civile, l'Église dispose «d'une juridiction propre et essentielle» que Jésus-Christ lui a donnée «qui consiste principalement dans le droit de faire des loix et des règlemens essentiel à toute société $»^{43}$. Selon lui, sur les deux champs d'action réservés à l'Église (la morale et les dogmes) la « puissance civile ne peut influer en rien $»^{44}$. Aussi, le texte de la Constitution civile est illégal car les députés ont statué sur la religion qui n'était pas de leur ressort. Pour lui comme pour de nombreux écrivains ecclésias-

(39) Le Journal chrétien, $\mathrm{n}^{\circ} 7$, p. 97-101.

(40) Décret de pacification proclamé par le Concile national de France dans l'église métropolitaine de Notre-Dame de Paris, 24 septembre 1797.

(41) Monique Cottret, «Les Jansénistes juges de Jean-Jacques », dans Jansénisme et Révolution, Actes du Colloque de Versailles, 13 et 14 juillet 1989, réunis par Catherine MAIRE, Paris, Chroniques de Port-Royal, 1990, p. 81-102.

(42) La Bible d'à présent puis ou des septante, dénonce dans son premier numéro, en 1790, « les députés qui n'ont pas d'expérience »,p. 4 et sur qui flotte « en vérité, un nuage de discrédit [qui] enveloppe les 1200 rois qui gouvernent la France », p. 5, BNF 8 Lc2 2345.

(43) Annales religieuses, politiques et littéraires, tome 1, n 9, p. 410, BNF 8 Lc2 884.

(44) Ibid., p. 409. 
tiques, « la religion » ne peut être " discutée comme la philosophie, avec la raison $»^{45}$. Ainsi, en respectant cette thèse, les journaux traditionnels accusent-ils la Constitution religieuse d'être " hérétique $»^{46}$ et les nouveaux prêtres des « intrus » anathématisés dont les sacrements sont nuls. Les journaux n'hésitent pas à livrer des témoignages de la France entière qui montrent le retour des prêtres apostats dans la bergerie (en rétractant leur serment) et le réveil des fidèles. Cette vision perdure après 1795 alors que la Constitution civile n'existe plus. Cependant, la confusion est encore grande chez les fidèles face aux deux clergés qui cohabitent. La presse catholique lance des annonces pour rassembler les prêtres, avec (pour les traditionnels) ou sans conditions (ou presque, pour les constitutionnels). Par ailleurs, en ces temps de reconquête religieuse, les journaux traditionnels hésitent quant au régime à plébisciter : Les Annales catholiques tentent de rassurer les lecteurs sur la capacité de la religion catholique à fonctionner aussi bien dans une République que dans un autre type de gouvernement. Toutefois, le seul qui possède un pouvoir omnipotent sur les lois de l'Église de France reste « le chef de l'Église universelle [le pape] qui décide ce qui convient de mieux dans ce temps de trouble et de guerre $»^{47}$.

\section{Le pape et le serment}

Rien ne semblait présager ce " schisme » jusqu'à l'obligation pour les ecclésiastiques de prêter un serment «à la nation, à la loi et au roi » en novembre 1790. La foi se politise ${ }^{48}$, accélère les prises de positions et les difficultés ${ }^{49}$ et provoque la division de la France : une partie soutient le clergé « assermenté » ou « jureur » (entre 52 et $55 \%$ ) et une autre défend le clergé non assermenté appelé également « réfractaire » ou insermenté. Cela entraîne un clivage durable, brisant l'harmonie de façade maintenue tant bien que mal depuis «l'année heureuse » de $1789^{50}$. Alors que l'As-

(45) Le Journal ecclésiastique, février 1790, p. 135, 139, 153.

(46) Annales religieuses, politiques et littéraires, tome 1, n 9, p. 412.

(47) Ibid., p. 417.

(48) Bernard Plongeron, « La modernité, enfant des révolutions », p. 304, dans Jean-Marie Mayeur, Charles Pietri, Luce Pietri, Bernard Plongeron (dir.), Histoire du christianisme, Les défis de la modernité (1750-1814), tome 10, Paris, Desclée, 1997.

(49) Albert Soboul écrit que l'attitude du pape « jeta le trouble dans les consciences et la France dans le schisme et la guerre civile », p. 204, dans La Révolution française, Paris, Gallimard, 1982. 1986 , p. 50

(50) Timothy Tackett, La Révolution, l'Église, la France, le serment de 1791, Paris, Cerf, 
semblée constituante avait proposé au pape l'honneur de «baptiser la Constitution civile », le premier pontife y perdait l'intégralité de ses prérogatives $^{51}$. S'appuyant sur le nouveau règlement, la presse constitutionnelle place l'obéissance à la loi et au pouvoir civil au-dessus des prérogatives de Rome. Une minorité de journalistes anticipent la réponse du pape en réclamant la tenue d'un concile ${ }^{52}$. Les deux brefs du 11 mars (Quod aliquantum) et du 13 avril 1791 (Caritas) qui condamnaient les principes de la Révolution et la Constitution civile arrivèrent trop tard, puisque l'obligation de prêter le serment « de fidélité à la Constitution du royaume et donc à la Constitution civile du clergé », le 27 novembre de l'année précédente, entraîna le schisme dans le clergé. Cette séparation n'était pourtant pas voulue et elle devient réelle dans les colonnes des journalistes où l'hostilité dépasse les simples mots.

Les feuilles constitutionnelles précisent avant tout que le serment n'est pas un acte religieux mais bien un engagement civil lors des élections. Ce mode de désignation ne rencontre pas l'assentiment des journalistes traditionnels qui vont systématiquement dénigrer les nouveaux « élus » comme des individus de la pire espèce des « grimauds, pelés, rustres, galeux, ignorans, ribauds crapuleux $\rangle^{53}$. Quant aux journaux opposés, ils qualifient les prêtres insermentés de «monstres» en leur enjoignant de s'éloigner « des autels », car ils n'y sont « plus digne[s] d'y paraitre $»^{54}$. Certains auteurs traditionnels dévaluent ces élections en arguant de forts taux d'abstentions. Aussi, à leurs yeux, «les intrus» ne sont attirés que par l'appât du gain et des revenus avant de se soucier de guider les âmes des fidèles ${ }^{55}$. D'autres ne dénigrent pas mais rappellent un élément de droit : le serment est possible mais il doit intégrer une limite concernant les aspects religieux avec « une réserve du spirituel ». L'Ami $d u$ roi publie chaque jour en dernière page, pendant près d'un an et demi, des professions de foi (de laïcs et de clercs) qui le plus souvent sont accompagnées de rétractations du serment : «Comme citoyen je dois le

(51) Le pape conserve sa primauté spirituelle sur l'Église mais il perd sa juridiction, les brefs sont soumis à la censure du gouvernement. Il n'a plus l'institution canonique des évêques, ces derniers, une fois élus, se contentent de lui envoyer un courrier pour le prévenir.

(52) Le Journal chrétien, ${ }^{\circ}$ 32, 7 avril 1792 : « Les conciles sont nécessaires pour maintenir l'unité entre les églises, la pureté de la doctrine, la sainteté des mœurs, la rigueur de la discipline et les libertés des églises », p. 503.

(53) Les Actes des Apôtres, chapitre 252, p. 12, BNF 8 Lc2 273.

(54) Le catéchisme républicain ou la France sauvée par l'abolition des rois et de la royauté, op. cit., p. 23.

(55) Selon la loi, tout curé devait recevoir un traitement minimal de 1200 livres, 1700 pour les vicaires, 25000 au maximum pour un évêque. 
serment de fidélité à ma patrie, comme chrétien je dois ma profession de foi ». Leurs auteurs déplorent leur faiblesse, la peur des menaces et implorent le pardon pour retourner dans le troupeau, témoignant de la véritable crise personnelle qui les a assaillis au moment du choix ultime. Elle signale les persécutions dont sont victimes les fidèles, obligés d'accepter « l'autre » et jouent sur la corde sensible pour apitoyer les lecteurs afin de soutenir les prêtres réfractaires. Ce combat théologico-juridique nuit probablement à la compréhension des articles et tous les journaux proposent des suites sur plusieurs numéros afin de ne pas lasser le public.

Les journaux constitutionnels adoptent un discours optimiste et sécurisant. Ils défendent les nouveaux prêtres. La loi les reconnaît, ce qui n'est pas toujours le cas des fidèles qui mettent en doute leur aptitude à pratiquer les sacrements. Dans le courrier des lecteurs, les rédacteurs montrent que tout se déroule pour le mieux (citant le nombre des jureurs par villes ou départements) mais ils ne passent pas sous silence leurs difficultés. Afin de convaincre les populations et de contrer les arguments du clergé réfractaire revigoré par la publicité de ces échecs - qui annonce aussi le nombre des réfractaires ou rétractés -, Le Journal chrétien propose de courts articles pour le "peuple et [les] gens de campagne » sur les bienfaits de la Constitution civile du clergé ${ }^{56}$, ou encore dans son numéro du 21 décembre 1791, le « Catéchisme sur la constitution civile du clergé par M. Molinier, prêtre de la doctrine chrétienne, évêque de Tarbes, Hautes-Pyrénées ». Il ajoute que cette brochure est « recommandable à tous les prêtres fidèles à la loi ». Après la Terreur, devant les difficultés persistantes pour le clergé assermenté, Les Annales de la religion rappellent les saines lectures pour renouer les liens entre le prêtre jureur et ses ouailles ${ }^{57}$.

\section{Un citoyen-chrétien ou un chrétien-citoyen?}

Les réformes entreprises ont considérablement troublé l'identité des catholiques. Comment peuvent-ils utiliser les nouveaux droits de citoyen et de croyant? Peuvent-ils le faire sans ambiguïté et sans conflit intérieur? Selon l'expression de Bernard Plongeron, n'y a t il pas d'autres

(56) Journal chrétien, ou l'Ami de la vérité et de la paix, $\mathrm{n}^{\circ}$ 4, 31 décembre 1791.

(57) Les Annales de la religion, $\mathrm{n}^{\circ} 14,1^{\mathrm{er}}$ août 1795 : «Apologie des prêtres français que l'amour de la religion et de la patrie a déterminé à accepter en 1791 la Constitution civile du clergé et en 1792 à prêter le serment de la Liberté et de l'Égalité prescrit par l'Assemblée nationale et la Convention nationale », BNF 8 Lc2 10. 
choix pour les insermentés entre « la valise ou le cercueil » ${ }^{58}$ ? Avant août 1792, les rédacteurs patriotes souhaitent, " autant qu'il est possible», "réparer les maux causés par l'abus de religion $»^{59}$ et guider à nouveau le peuple des fidèles grâce aux nouvelles lois religieuses vers la « morale », « le bonheur général $»^{60}$ et le triomphe sur le fanatisme. Les journaux se fixent pour objectif d' $\mathrm{d}^{\prime}$ éclairer le peuple sur ces véritables devoirs de citoyen et de chrétien » en démasquant la conduite de « ceux [qui] cherchent à tromper le peuple», et de montrer ensuite que la Constitution civile, « bien loin de porter atteinte à la religion, lui rend au contraire sa première splendeur $\rangle^{61}$. Ils stigmatisent le comportement des ministres des autels ${ }^{62}$, des moines (« ces hypocrites là ! ») $)^{63}$, des « pervers, couverts d'un manteau religieux » qui ne reconnaissent pas la Constitution civile comme nouvelle loi de l'Église. L'accepter comme citoyen devient une garantie de rester un bon chrétien. La laïcisation est en marche car dorénavant, la qualité de chrétien dépend entièrement de sa citoyenneté. Les journalistes modèlent le statut d'un citoyen-chrétien "parfait» à leurs yeux : en 1790 il doit être obéissant aux lois (y compris bien évidemment aux lois religieuses et prêter les serments), actif en participant à la vie politique (clubs, sociétés...), vertueux, généreux, rayonnant dans son milieu social ou familial. Les rédacteurs n'hésitent pas à montrer ces « catholiques irréprochables » en les dépeignant sous les traits du « pauvre généreux » ou du père de famille. Comme le « sans-culotte Jésus-Christ », le citoyen est animé par le désintéressement qui doit conduire également le gouvernement vers la société parfaite. On remarquera que les journaux n'évoquent quasiment pas le « décadi », la journée désignée pour valoriser les bons citoyens.

Les journalistes traditionnels défendent le principe de la tolérance religieuse qui doit préserver leur citoyenneté s'ils refusent le serment. Ils saluent, dans leurs colonnes, ceux qui font la publicité de leurs erreurs en abjurant leur serment ${ }^{64}$. Ce «bulletin» de bon citoyen (et chrétien)

(58) Michel Vovelle (dir), L'État de la France pendant la Révolution française, 1789-1799, Paris, La Découverte, 1988, p. 242.

(59) Correspondance religieuse et morale avec les départements, janvier-juillet 1792, BNF 8 Lc2 656 (Bis).

(60) Ibid., prospectus, 1791 p. 1 et 2.

(61) Journal des ecclésiastiques constitutionnels, prospectus, p. 2.

(62) Ibid., prospectus, janvier-avril 1792, BNF 8 Lc3 8.

(63) Le Journal du diable, octobre 1790, BNF 8 Lc2 359, n 1, p. 1.

(64) Les Annales religieuses, politiques et littéraires, tome 2, $\mathrm{n}^{\circ} 3$ : « Hâtez-vous donc Ô prêtres constitutionnels! D'attirer sur vous-même un jugement de miséricorde et de salut!", p. 128. 
s'applique également aux clercs. Après 1795, la volonté existe toujours de montrer l'importance d'être « chrétiens et français [...] un double lien qui fortifiera leur attachement à la religion et à la patrie $\rangle^{65}$. Après les temps difficiles de la Terreur, l'heure est à l'union entre les laïcs et les prêtres. La vision du «citoyen-chrétien " s'inscrit dans le contexte de la politique religieuse de la Convention thermidorienne et du Directoire. Les lois du 11 prairial de l'an III (30 mai 1795) et du 7 vendémiaire de l'an IV (29 septembre 1795) reconnaissent la liberté religieuse et l'exercice quasi libre du culte. En échange, un serment dit de « soumission » (le troisième serment depuis les débuts de la Révolution) aux lois de la République est exigé ${ }^{66}$. C'est en même temps un renouveau de l'Église catholique. Néanmoins, pour l'Église constitutionnelle, la concurrence existe toujours car les édifices sont parfois partagés avec les défenseurs de l'Église romaine. Il devient alors urgent de recruter les fidèles délaissés pendant la Terreur par un discours rassembleur, parfois racoleur, et les écrivains placent dans l'appartenance à la patrie, la base de tout ralliement, une modalité dont on ne peut échapper en ces nouveaux temps de paix. Les auteurs s'adressent bien évidemment à ceux qui n'ont pas prêté les serments et également à ceux qui se sont éloignés de la religion dans les temps difficiles. L'obéissance (en façade ?) aux lois est de rigueur et « tout catholique doit aux lois de la République, soumission sincère et véritable $»^{67}$.

De 1789 à 1799, des journaux affirment leur sensibilité et leur identité religieuses. Sans être nombreux, les périodiques entièrement consacrés à la défense de la religion se nourrissent des débats religieux pour marquer leurs différences. Pourtant, ces feuilles ont des difficultés pour se faire connaître en raison du ton élitiste et parfois peu intelligible de leurs articles (pour le commun des lecteurs). C'est sans doute pour cela que les journalistes sont aussi en majorité des ecclésiastiques. Les archives font défaut pour retrouver les listes d'abonnés et il est difficile de connaître l'audience des titres et de saisir la composition de leur lectorat. On connaît les chiffres avancés pour les journaux généralistes (10 000 abonnés pour Les Annales patriotiques de Linguet, 15000 pour Le Mercure de France $)^{68}$;

(65) Journal du Concile national de France, 11 fructidor an 5-28 août 1797, n 3, p. 17, $\mathrm{BNF} 8 \mathrm{Lc} 366\left(\mathrm{n}^{\circ} 1\right.$ à 22$)$.

(66) Bernard Cousin, Monique Cubells, René Moulinas, op. cit., « Je reconnais que l'univers des citoyens français est le souverain et je promets soumission et obéissance aux lois de la République », p. 212.

(67) Journal du Concile national de France, 11 septembre 1797, $\mathrm{n}^{\circ}$ 7, p. 51. 1984, p. 100 .

(68) Jean-Paul Bertaud, La presse et le pouvoir : de Louis XIII à Napoléon, Paris, Perrin, 
les journaux religieux en sont sans doute fort éloignés. En février 1792, Le Journal chrétien qui connaît des problèmes financiers avance le chiffre de 600 abonnés. En l'an VII, le ministère de la Police générale dresse une « nomenclature des journaux et feuilles périodiques qui ont circulé par la poste ». Les Annales de la religion compteraient 42 abonnés ${ }^{69}$. Enfin, Le Journal du Concile national de France aurait été diffusé à 300 exemplaires. Il faut bien évidemment rester prudent avec ces chiffres qui peuvent être éloignés de la réalité ; cependant, un certain nombre d'indices peut nous informer sur le poids réel des journaux religieux entre 1789 et 1799. Le succès d'un titre attire forcément l'attention de l'administration et de ses concurrents : les contrefaçons (c'est le cas pour Les Actes des Apôtres), les dissidences avec tentatives de captation des abonnés (le libraire Leclerc, diffuseur de journaux et d'ouvrages pour les deux clergés, tente en septembre 1795, d'utiliser le double de la liste des abonnés pour Le Journal de la religion et du culte catholique $)^{70}$, les agressions contre les journalistes et les libraires, ou encore la prohibition des titres en l'an $\mathrm{V}$ et VI (Les Annales catholiques sont supprimées le $1^{\text {er }}$ septembre 1797-15 fructidor an $\mathrm{V}$ et leurs auteurs condamnés à la déportation; Les Mémoires pour servir à l'histoire et à la philosophie continuateur des Annales de la religion sont prohibés en germinal an VI pour « fanatisme ») et enfin, la veille de sa disparition, Les Annales catholiques annoncent que « le journal et les ouvrages de M. de La Harpe sont fort courus » à Rome et que si la poste fonctionnait mieux le nombre d'abonnés augmenterait $^{71}$. Ainsi, la " presse religieuse catholique » gêne et est soumise à la même censure, ce qui prouve la portée supposée de ces feuilles dans le débat politique sous le Directoire ${ }^{72}$. Avec le serment, les journalistes catholiques doivent choisir un camp, ce qui les divise définitivement. C'est la première rupture pour le clergé et pour la " presse religieuse » en Révolution, une rupture politique et religieuse. Pour les journalistes de la presse traditionnelle, l'alliance passagère avec la presse royaliste les a desservis, car la figure du catholique les assimilera à celle du royaliste. Il faut dire qu'ils usent des mêmes contestations (l'antiphilosophie, l'usurpation des pouvoirs...). Les journaux constitutionnels souffriront aussi

(69) $A N$, F7 3448.

(70) Les Annales de la religion, $\mathrm{n}^{\circ} 20,12$ septembre 1795, p. 479.

(71) Les Annales catholiques, tome 4, $\mathrm{n}^{\circ} 42$, p. 199, BNF 8 Lc2 885-886.

(72) AN F7 3448, n $4685 ; n^{\circ} 3518 ; 18$ floréal an VI, dénonciation contre « Les Annales des religions » journal qui défend le « parti des prêtres catholiques », « tendance à rendre par tous les moyens licites la religion catholique une religion dominante dans la République ». 
d'avoir uni leur destin à un gouvernement qu'ils ont défendu et soutenu et qui a imposé la nouvelle loi religieuse. On assiste alors à la disparition de la " presse religieuse » jusqu'en 1794. La seconde rupture observée dans cette presse apparaît postérieurement. Les journaux évoquent moins directement les sujets politiques et disent respecter le nouveau régime. S'adaptant, en février 1795, à la loi qui proclame la neutralité religieuse de la République, constitutionnels et traditionnels limitent leur activité littéraire au soutien actif des prêtres réfractaires ou assermentés. Sans violence dans leurs mots, ils se heurtent à l'hostilité des Thermidoriens qui ne souhaitent plus intégrer la religion à l'État ${ }^{73}$. Pour chaque clergé, l'heure est à la réorganisation et au rassemblement, parfois contre un ennemi commun, les théophilanthropes, mais sans plan de bataille collectif.

Organisés autour de l'évêque constitutionnel Grégoire et de l'Imprimerie chrétienne, les évêques «constitutionnels» se servent des Annales de la religion pour « affermir le clergé patriote » et « restaurer le culte $»^{74}$. En l'an IV, il fait partie « des journaux rédigés et publiés à Paris et dont les rédacteurs ou propriétaires ont fait leur déclaration » sur un total de 116 feuilles de l'époque. Dans ses articles, il ne s'occupe que de religion et il relaie les thèmes chers aux évêques réunis : le statut de « citoyen-chrétien » (l'obéissance à la loi permet de conserver son identité de chrétien), la réunion avec les $"$ dissidents ${ }^{75}$ (les insermentés), les sacrements, l'installation des évêques et des curés dans les diocèses et les paroisses ainsi que leur mission pastorale, les élections et la désignation aux sièges vacants, l'éducation de la jeunesse par l'instauration des Écoles chrétiennes, par la Société du même nom ${ }^{76}$, les correspondances avec les Églises étrangères et notamment celles des colonies ${ }^{77}$ et l'organisation du

(73) Jean-René Suratteau, «Le Directoire avait-il une politique religieuse ?», AHRF, $\mathrm{n}^{\circ} 283$, p. $79-92$.

(74) Compte-rendu par le citoyen Grégoire au Concile national des travaux des Évêques réunis à Paris, imprimé par ordre du Concile national, 84 pages et AN F7 3448, « lettres, rédacteurs, registres, abonnement, police des journaux ». À noter que parmi ces 116 journaux qui ont fait leur déclaration devant le ministère de la Police générale, nous comptons seulement trois journaux mentionnant dans leur titre la religion : Les Annales de la religion, La Feuille hebdomadaire du Concile national et Le Journal du Concile national de France.

(75) Décret de pacification proclamé par le Concile national de France dans l'église métropolitaine de Notre-Dame de Paris, dimanche 24 septembre 1797 (3 vendémiaire an VI de la République française).

(76) Lettre synodique du Concile national de France aux pères et mères et à tous ceux qui sont chargés de l'éducation de la jeunesse, 23 pages.

(77) Compte-rendu par le citoyen Grégoire... op. cit., p. 58 et Rapport concernant la religion dans les colonies du nouveau monde fait au Concile national par un de ses membres, 12 pages. 
futur Concile national prévu pour le mois d'août 1797. C'est un programme ambitieux qui souffre de la renaissance du culte romain, dont la publicité est faite par Les Annales catholiques (ex-Annales religieuses, politiques et littéraires). Tirant à boulets rouges sur les constitutionnels, Les Annales catholiques réfléchissent aux procédures de pénitence pour ramener l'unité de l'Église tout en décriant le projet d' « un concile incognito qui s'intitule effrontément national quand l'immense majorité du clergé français ni ne le connoît, ni ne peut le connaître $\gg^{78}$.

Avec la suppression de la Constitution civile en septembre 1794, les députés n'ont pas répondu au problème de la foi pour un citoyen. Dans l'attente de réunir les déçus et les indécis, la survie de la religion catholique passe par le retour dans la « bergerie $»^{79}$ des populations rurales ${ }^{80}$. Ce calme apparent est pourtant contraire à l'image donnée du catholique (surtout le réfractaire) qui complote sans cesse dans l'ombre. Faut-il donner un crédit à cette image ? Les nombreuses dénonciations contre certains «journaux religieux »- qui ont fait l'objet de procédures de surveillance et de prohibition - nous prouvent le contraire. Pourtant, les « feuilles religieuses » se font de plus en plus rares jusqu'en 1799. Certes, elles peuvent changer de titres ou être absorbées par d'autres journaux, mais on ne peut que constater certaines absences et une activité limitée de leur part : elles chicanent sur les conflits d'installation des évêques, mais ne participent pas aux campagnes électorales (c'était déjà le cas en 1790) ; les femmes, d'ordinaire très actives dans la défense de la religion sont peu mentionnées dans les articles. De même, les journaux réagissent peu aux autres débats culturels et scientifiques de l'époque à l'exception des projets éducatifs, mais qui restent à l'état d'ébauche. Faut-il y voir réellement une distance progressive du « journaliste catholique » dans la citoyenneté politique et dans la société ? Fuit-il les risques de censure en «oubliant» certains thèmes de combat? L'importance grandissante de la rubrique qui annonce les publications d'ouvrages montre pourtant le contraire. Les journalistes s'adaptent et déplacent le combat des mots sur ce terrain littéraire, qui est complémentaire de l'entreprise de presse. Selon Albert Soboul, «l'art littéraire se met au

(78) Annales catholiques, $\mathrm{n}^{\circ}$ 41, tome 4, BNF, 8 Lc2 886, page 186.

(79) Collection des pièces imprimées par ordre du Concile national de France, 343 p. 1797, BNF Ld4 461.

(80) Lettre synodique, le Concile national de France aux pasteurs et aux fidèles sur les moyens de rétablir la paix religieuse, $23 \mathrm{p}$. 
service de l'action $»^{81}$ et les hommes de lettres (tels Volney, Chateaubriand, de Maistre, de Bonald) font " pousser » hors du journal les polémiques religieuses et politiques. La "presse religieuse et catholique» attend sûrement un temps plus propice où ses discours, malgré les réformes et les disputes rencontreront l'assentiment de la population et des législateurs.

Guillaume CoLOT

Doctorant, Centre d'histoire « Espaces et cultures » Université Blaise-Pascal (Clermont II) 4 , rue Ledru 63057 Clermont-Ferrand Cedex 1 colot@caramail.com 


\section{Annexe : Liste des journaux dont le titre ou l'auteur évoque la religion, d'après Walter-Martin}

\section{Nom du journal}

A deux liars, a deux liars, le Journal

(abbé de Bouyon) (oct 1791- juillet 1792)

L'almanach de l'abbé Maury (1790-1791)

Les Actes des Apôtres (1791-1792)

Actes des Apôtres et des martyrs (1796)

Actes des bons apôtres, journal des disciples

de la divinité française, c'est-à-dire de la nation,

de la loi et du roi (juin 1790)

Actes des capucins (avril 1790)

Actes des martyrs (mai 1797)

L'ami de la religion, des mours et des sciences (août 1797)

L'ami des vieillards, Journal de la bienfaisance $3 \mathrm{vol}$

(1791)

L'ami du clergé et de la noblesse (1791)

L'ange Gabriel

Annales de la religion (1794-1800)

Annales religieuses, politiques et littéraires

(1795-1797)

Bible d'à présent (1790)

Catéchisme à l'usage des gouvernans et des gouvernés (an III)

Catéchisme de la république française

ou conférence curieuse entre un citoyen

du faubourg Saint-Antoine et un modéré

de la ci-devant AN (1792)

Catéchisme républicain (an II)

Correspondance religieuse et morale

avec les départements (1791-1792)

Disciple des apôtres (1789)

Catéchisme de Jean Bart (1790)

Journal chrétien (1791-1792)

Journal du Concile national de France (1797)

Journal de la noblesse, de la magistrature,

du sacerdoce et du militaire (1790-1791)

Journal de la religion

Journal de la religion et du culte catholique

(1795)

Journal des ecclésiastiques (1792)

Courrier du midi (1792)

Journal de la vente des $B N$

Journal des prêtres et des nouvelles

ecclésiastiques

Courrier de Paris, journal des primes

\section{Nombre de volumes Côtes (BNF)} ou de nos

2 vol

$8^{\circ} \mathrm{Lc}^{2} .647(\mathrm{~A}, \mathrm{~B})$

$11 \mathrm{vol}, 311 \mathrm{n}^{\circ}$

$8^{\circ} \mathrm{Lc}^{2} .2359$

4 vol

$3 n^{\circ}$

$8^{\circ} \mathrm{Lc}^{2} .273$

$8^{\circ} \mathrm{Lc}^{2} .921$ et 922

$8^{\circ} \mathrm{Lc}^{2} .2362$

$2 n^{\circ}$

$3 n^{\circ}$

$8^{\circ} \mathrm{Lc}^{2} .3305$

$8^{\circ} \mathrm{Lc}^{2} .952$

$4^{\circ} \mathrm{Lc}^{2} .2675$

$8^{\circ} \mathrm{Lc}^{2} .616-620$

Prospectus
$7 \mathrm{n}^{\circ}$
18 vol
$3 \mathrm{vol}$

$8^{\circ} \mathrm{Lc}^{2} .254$

$8^{\circ} \mathrm{Lc}^{2} .2754$

$8^{\circ} \mathrm{Lc} 3.10$

$8^{\circ} \mathrm{Lc}^{2} .885-886$

Prospectus, $4 \mathrm{n}^{\circ}$

$1 \mathrm{n}^{\circ}$

$8^{\circ} \mathrm{Lc}^{2} .2345$

$8^{\circ} \mathrm{Lc}^{2} .2632$

$1 n^{\circ}$

$8^{\circ} \mathrm{Lc}^{2} .2552$

$3 n^{\circ}$

2 vol

$8^{\circ} \mathrm{Lb} 41.2284$.A

$8^{\circ} \mathrm{Lc}^{2} .656$

Prospectus, $6 \mathrm{n}^{\circ}$

$8^{\circ} \mathrm{Lc}^{2} .275$

(microfilm

m.4233 (10)

$2 \mathrm{n}^{\circ}$

$8^{\circ} \mathrm{Lc}^{2} .375$

3 vol

$8^{\circ} \mathrm{Lc}^{2} .625-627$

4 vol

$8^{\circ} \mathrm{Lc}^{2} .492$

Prospectus

$12 \mathrm{n}^{\circ}$

$8^{\circ} \mathrm{Lc}^{2} .641$

$8^{\circ} \mathrm{Lc}^{2}$.884.bis

Prospectus, $5 \mathrm{n}^{\circ} \quad 8^{\circ} \mathrm{Lc} 3.8$

$4^{\circ} \mathrm{Lc} 3.9$

6 vol

$8^{\circ} \mathrm{Lc}^{2} .470$

Prospectus, $3 \mathrm{n}^{\circ} \quad 4^{\circ} \mathrm{Lc}^{2} .287$

$4^{\circ} \mathrm{Lc}^{2} .988$ 


\[ \text { Nom du journal } \]
Journal des théophilanthropes
Journal du diable (1790)
Journal ecclésiastique (1789-1790)
Journal religieux ou mémoires pour servir
à l'histoire de l'Église (1798)
Juif errant (1799)
Juif errant (lettres)
Le prêcheur populaire
Le précurseur du messie
Prônes civiques ou le pasteur patriote (1791)
Les quatre évangélistes (1790)
Le tonneau de Diogène ou les révolutions
du clergé (1790)
Nouvelles ecclésiastiques
Les loisirs d'un curé déplacé ou les actes
de l'Église constitutionnelle (1791)

Nombre de volumes Côtes (BNF) ou de nos

$19 n^{\circ}$

$84 \mathrm{n}^{\circ}$

91 vol (10 derniers

$\mathrm{RF}$ )

$8 n^{\circ}$

$3 n^{\circ}$

Prospectus

2 vol

$5 \mathrm{n}^{\circ}$

4- $12 \mathrm{n}^{\circ}$

$33 n^{\circ}$

$8^{\circ} \mathrm{Lc}^{2} .995$

$8^{\circ} \mathrm{Lc}^{2} .359$

H8810-20

$8^{\circ} \operatorname{Lc} 3.67$

$8^{\circ} \mathrm{Lc}^{2} .2346$

$8^{\circ} \mathrm{Lc}^{2} .2727$

$8^{\circ} \mathrm{Lc} 2.321$

$8^{\circ} \mathrm{Lc}^{2} .1006$

$8^{\circ} \mathrm{Lc}^{2} .555$

$8^{\circ} \mathrm{Lc}^{2} 273$.bis

$8^{\circ} \mathrm{Lc}^{2} 311-\mathrm{A}$

$26 \mathrm{vol}$

$4^{\circ} \mathrm{Lc} 3.2$

2 vol

$8^{\circ} \mathrm{Lc}^{2} .634$ 